\title{
Large scale synthesis and characterization of Ni nanoparticles by solution reduction method
}

\author{
HUAZHI WANG*, XINLI KOU, JIE ZHANG and JIANGONG LI \\ Institute of Material Science and Engineering, Lanzhou University, Lanzhou 730000, P R China
}

MS received 1 May 2007

\begin{abstract}
Ni nanoparticles were mass synthesized by solution reduction process successfully. The influence of the parameters on the particle size of Ni nanoparticles were studied and the referential process parameters were obtained. The morphology and structure of the synthesized Ni nanoparticles were characterized by scanning electron microscopy (SEM), X-ray diffraction (XRD), Brunauer-Emmett-Teller (BET) surface area analysis and infrared spectroscopy (IR). The results show that Ni nanoparticles are of high purity and are covered by hydroxyethyl carboxymethyl cellulose (HECMC) layer and the mean size being about $31 \mathrm{~nm}$. The magnetic measurement revealed that $\mathrm{Ni}$ nanoparticles are ferromagnetic.
\end{abstract}

Keywords. Nanomaterials; microstructure; magnetic properties.

\section{Introduction}

With the development of nanomaterials and nanotechnology, more and more efforts have been directed toward large-scale synthesis of nanoparticles in recent years due to their potential applications in many areas (Guo et al 2006; Liu Q et al 2006; Luo et al 2006; Wei et al 2006). In such uses, nanoparticles with different purity, size, shape and structure will greatly influence the ultimate performance of the devices, accordingly, preparation of nanoparticles with desired quality and low cost by convenient method in large scale is of great importance.

As an important transition metal, Ni nanoparticles have wide ranging applications in the fields of permanent magnets, magnetic fluids, magnetic recording media, solar energy absorption, fuel cell electrodes, catalysts etc. So the synthesis of Ni nanoparticles has attracted considerable attention. Although many methods are used to prepare $\mathrm{Ni}$ particles (Pfeil and Leonard 1987; Degen and Matek 1999; Zheng et al 2001; Ni et al 2003; Syukri et al 2003; Hou and Gao 2004; Kumar et al 2004; Liu et al 2006), only a few of them are used in its large scale synthesis. For example, Wei and co-workers (2006) prepared Ni nanoparticles in large scale using an anodic arc plasma technique and Pfeil and co-workers (1987) prepared Ni powder by decomposition of nickel carbonyl. To our knowledge, there are only a few reports of Ni nanoparticles produced by solution reduction method in large scale till now.

As a nanomaterial synthesis technique, solution reduction method has many advantages as compared with other

*Author for correspondence (wanghuazhi@lzu.edu.cn) methods (Degen and Matek 1999; Zheng et al 2001). It is a convenient, inexpensive and easy to handle technique. In our laboratory, this method was developed to mass synthesize Ni nanoparticles, and the goal has been achieved.

The purpose of this paper is to describe a facile way for preparing Ni nanoparticles in large scale by the solution reduction process. Pure Ni nanoparticles have been obtained and characterized herein.

\section{Experimental}

Ni nanoparticles were synthesized through a solution reduction process using hydrate hydrazine as a reducing agent. First, $\mathrm{HECMC}$ was added to $\mathrm{NiCl}_{2} \cdot 6 \mathrm{H}_{2} \mathrm{O}$ solution with a weight $\%$ of $0 \cdot 2$. Then $\mathrm{NaOH}$ solution and hydrate hydrazine was added into the above mixture until the $\mathrm{pH}$ value was about 11 . The resulting solution was kept in a thermostatic bath until black powder precipitated completely. Finally, the product was washed with distilled water and ethanol for several times, and then dried in a vacuum drying oven at room temperature.

The morphology of the particles was observed on a HITACHI S-4800 scanning electron microscope with an acceleration voltage of $30 \mathrm{kV}$. The specific surface area of Ni particles was measured by nitrogen sorption isotherms at $77 \mathrm{~K}$. The crystal structure of the sample was analysed by X-ray diffraction on a Rigaku D/max-2400 diffractometer with $\mathrm{CuK}_{\alpha}$ radiation. The background was removed in the intensity determination. The $\mathrm{K}_{\alpha_{2}}$ diffraction peaks were eliminated by the improved Rachinger's method. Silicon powder with a purity of $99.9999 \%$ was used as an external standard material to calibrate the peak positions. The positions of the observed diffraction peaks were ob- 
tained by a centre of gravity method. For the calculation of the lattice constant of the Ni nanoparticles, the X-ray powder diffraction patterns were recorded in a range from $35-105^{\circ}$ with a step width of $0.02^{\circ}$ and a counting time of $6 \mathrm{~s}$ per step. Infrared spectrum on $\mathrm{KBr}$ pellets was recorded on a NICOLET NEXUS 670 FT-IR spectrophotometer. The magnetic properties of the sample was studied by a Lake Shore 7304 vibrating sample magnetometer at room temperature.

\section{Results and discussion}

\subsection{Influence of parameters on size of Ni nanoparticles}

It is known that, in the process of synthesizing monodispersed particles, there are two steps: nucleation step and growth step (La Mer and Dinegar 1950; Liu Q et al 2006). These two steps depend on the reaction system and the parameters controlling the reaction. In our experiment, with the concentration of the reactants and the synthesis temperature increased, the reaction kinetics would be enhanced (Hinotsu et al 2004). So the nucleation of Ni crystals would be accelerated, and more nucleus of Ni crystals are formed. With the more Ni nucleus formed, the collision and coalescence rates of Ni nucleus would get higher. Therefore, in the process of synthesizing $\mathrm{Ni}$ nanoparticles, the effects of the concentration of the reactants and the synthesis temperature on the particle growth may be explained by considering two competing aspects: first, with the temperature and the concentration of the reactants increased, the number of Ni nuclei would also be increased, which would ordinarily leads to the decreased size of Ni nanoparticles. On the other hand, higher temperature and concentration of the reactants would lead to higher rate of coagulation to result in greatly enhanced opportunity for the nuclei to grow into larger particles. Therefore, the size of $\mathrm{Ni}$ nanoparticles would not be simply increased or decreased with the increase of concentrations of $\mathrm{NiCl}_{2} \cdot 6 \mathrm{H}_{2} \mathrm{O}$ and the synthesis temperatures. In our work, when the concentration of $\mathrm{NiCl}_{2} \cdot 6 \mathrm{H}_{2} \mathrm{O}$ is $0.8 \mathrm{M}$ and the synthesis temperature is $80^{\circ} \mathrm{C}$, respectively, we would synthesize $\mathrm{Ni}$ nanoparticles with the mean size of about $31 \mathrm{~nm}$.

\subsection{Microstructure}

The SEM observations of the sample (figure 1) reveal that the shape of the Ni particles is nearly spherical and the distribution of the particles (see figure 2) is relatively narrow. From figure 1, we can determine the average diameter of the sample to be about $31 \mathrm{~nm}$, and we can also see a certain extent of aggregations of the particles which might have been resulted from magnetic interaction and polymer adherence between the particles.

The surface area of Ni particles was determined using BET technique. The theoretical particle size was calcu- lated from surface area, assuming spherical particles, from the equation

$$
D_{\mathrm{BET}}=6000 /(\rho S) \text {, }
$$

where $D_{\mathrm{BET}}$ is the equivalent particle diameter in nanometers, $\rho$ the density of the particle in $\mathrm{g} / \mathrm{cm}^{3}$ and $S$ the specific surface area in $\mathrm{m}^{2} / \mathrm{g}$. From BET analysis, we obtained the specific area as $16.53 \mathrm{~m}^{2} / \mathrm{g}$, so the average equivalent particle size is $40 \mathrm{~nm}$, which is slightly larger than the result deduced from SEM observation. This difference may have been aroused from the aggregation of the nanoparticles which may make the average equivalent particle size deduced from BET technique larger than it actually is.

The crystal structure of the sample was analysed by XRD. Figure 3 shows the XRD pattern of the sample. It can be seen that it is the typical diffraction pattern of a

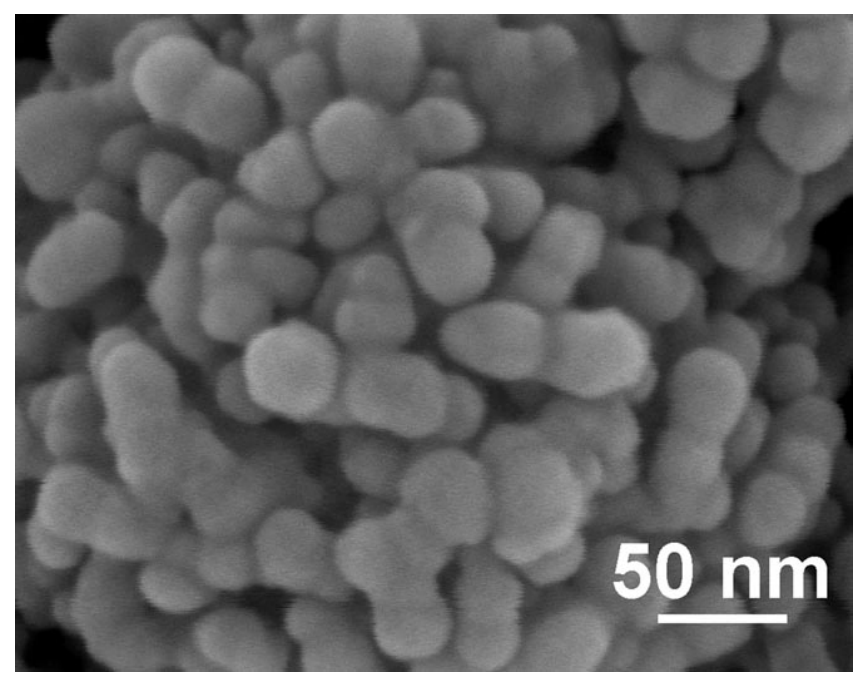

Figure 1. SEM image of the sample.

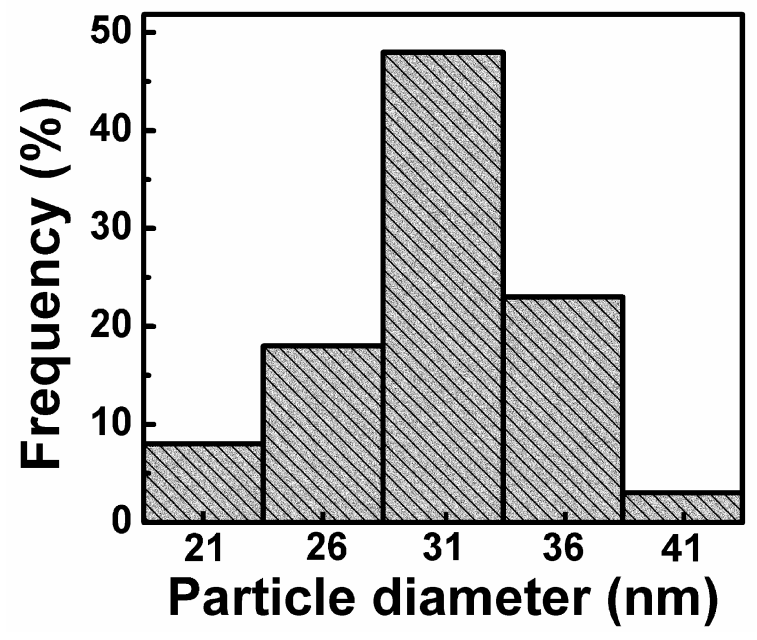

Figure 2. Particle size distribution of the sample. 
face-centred cubic phase without any other additional peaks, indicating that the $\mathrm{Ni}$ nanoparticles are singlephased $f c c$ phase. The broad diffraction peaks are indicative of the ultrafine Ni particles.

Lattice constant of the Ni particles has been calculated accurately from the observed diffraction peaks by a program based on Cohen's analytical least squares refinement. The lattice constant of the $\mathrm{Ni}$ nanoparticles was calculated to be $3.524 \AA$. It is slightly larger than the corresponding bulk value of $3.523 \AA$ (Swanson and Tatge 1953). This is abnormal for it was anticipated that the lattice constant of a nanoparticle would be smaller than the bulk value according to the point of view of $\mathrm{Yu}$ and co-workers (1999). They reported the lattice contraction of the $\mathrm{Sn}$ and $\mathrm{Bi}$ nanoparticles with decreasing particle size. To make this abnormal phenomenon clear, we recorded IR spectrum of the Ni nanoparticles. IR spectrum of the Ni nanoparticles is shown in figure 4. From the spectrum, we observe that this organic component has vibration peaks at $1642 \mathrm{~cm}^{-1}, 1018 \mathrm{~cm}^{-1}, 3422 \mathrm{~cm}^{-1}$ and $2909 \mathrm{~cm}^{-1}$, indicating the existence of carboxymethyl, hydroxyl and hydroxyethyl. This analysis indicates that the Ni nanoparticles are covered by a HECMC layer completely. We considered the abnormal phenomenon, that the measured lattice constant of the sample is slightly larger than the corresponding bulk value, may probably be caused by the polymers on the surface of the particles, for surface adsorption would lead to lattice expansion (Törnqvist et al 1984; Sun 2003).

It is well known that nanometer sized $\mathrm{Ni}$ particles get easily oxidized in air. However, after the sample was exposed in air for six months, we found that except for pure $f_{c c}-\mathrm{Ni}$, no Ni oxide phase was detected. This means that the polymer layer on the surface of the sample has a strong ability of protecting itself from being oxidized.

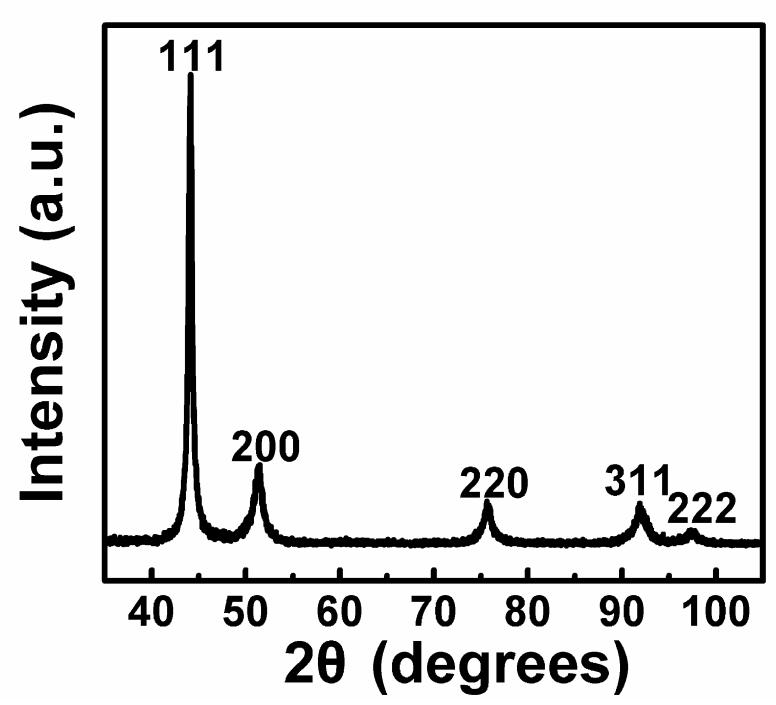

Figure 3. XRD pattern of the Ni nanoparticles

\subsection{Magnetic properties}

Magnetic properties of the sample were investigated at room temperature using a vibrating sample magnetometer with an applied field, -6000 Oe $\sim 6000$ Oe. Figure 5 shows the hysteresis loop of the sample which is a typical ferromagnet. The saturation magnetization of the sample is about $43 \mathrm{emu} / \mathrm{g}$ measured above $50 \mathrm{kOe}$. This value is approximately the same as also reported by Hou and Gao (2004), but it is lower than that of the pure $f c c-\mathrm{Ni}$ phase in bulk form (54.39 emu/g) (Gray 1972), which may be caused by the presence of the polymer layer on the surface of the nanoparticles.

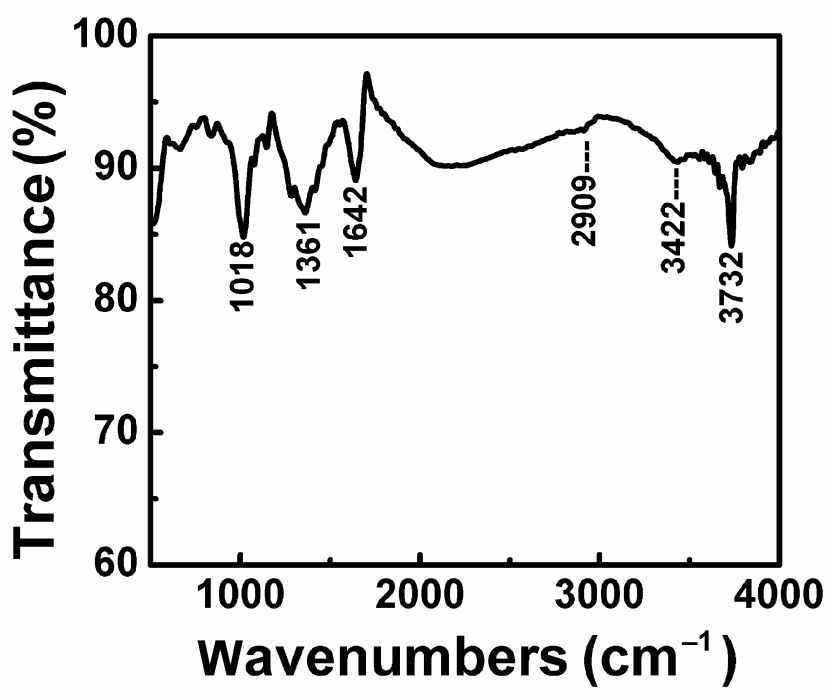

Figure 4. IR spectra of the Ni nanoparticles.

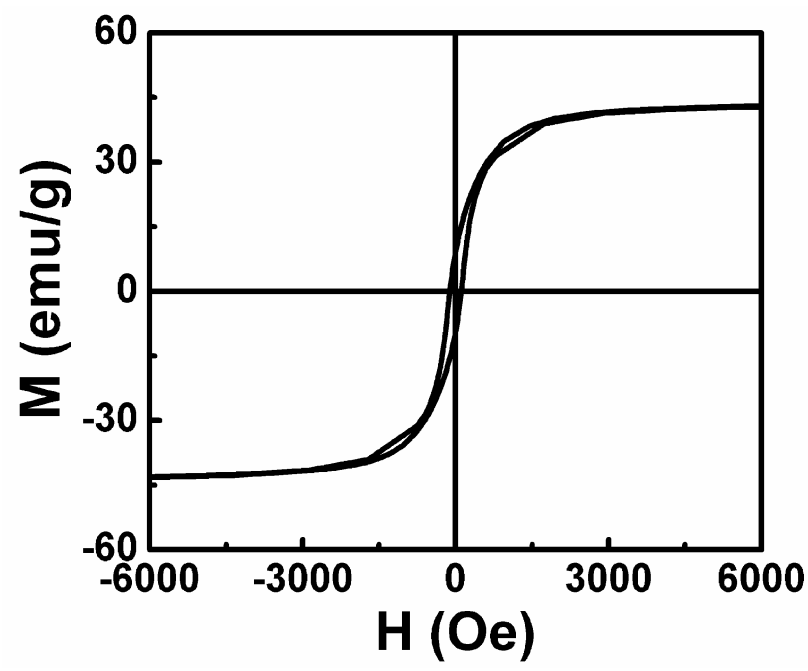

Figure 5. Hysteresis loop of the $\mathrm{Ni}$ nanoparticles at room temperature 


\section{Conclusions}

(I) $\mathrm{Ni}$ nanoparticles were successfully synthesized by solution reduction method for mass production. The synthesized Ni nanoparticles were highly pure and homogenous in size. For its simpleness, easy controllability and low cost, solution reduction method is believed to have potential applications in synthesizing other metals in high yield. Further research is under progress in our laboratory. (II) The crystal structure of the sample is $f c c$ structure, the specific surface area is $16.53 \mathrm{~m}^{2} / \mathrm{g}$. The mean particle size, $31 \mathrm{~nm}$, obtained from SEM observation.

(III) Due to the presence of HECMC on the surface of the particles, the lattice constant of Ni nanoparticles is larger than the bulk value; the saturation magnetization of the sample is lower than the bulk value.

\section{References}

Degen A and Matek J 1999 NanoStruct. Mater. 12225

Hinotsu T, Jeyadevan B, Chinnasamy C N, Shinoda K and Tohji K 2004 J. Appl. Phys. 957477

Hou Y L and Gao S 2004 J. Alloy. Compd. 365112

Gray D E 1972 America institute of physics handbook (New York: McGraw-Hill Book Company)
Guo P S, Sun Z, Chen Y W and Zheng Z H 2006 Mater. Lett. 60966

Kumar D, Yarmolenko S, Sankar J, Narayan J, Zhou H and Tiwari A 2004 Compos. Part B-Eng. 35149

La Mer V K and Dinegar R H 1950 J. Am. Chem. Soc. 724847

Liu Q, Liu H J, Liang Y Y, Xu Zh and Yin G 2006 Mater. Res. Bull. 41697

Liu X S, Meridor U, Zhao P, Song G B, Frydmanc A and Gedanken A 2006 J. Magn. Magn. Mater. 30113

Luo Zh S, Chen Ch L, Zhao D J, Luo Sh L and Li Zh Ch 2006 Chem. Phys. Lett. 421584

Ni X M, Su X B, Yang Z B and Zheng H G 2003 J. Cryst. Growth 252612

Pfeil and Leonard B 1987 US Patent 4,673,430

Sun C Q 2003 Prog. Mater. Sci. 48521

Swanson H E and Tatge E 1953 Nat. Bur. Stand. (US) Circ. 539 I 13

Syukri T Ban, Ohya Y and Takahashi Y 2003 Mater. Chem. Phys. 78645

Törnqvist E, Adams E D, Copel M, Gustafsson T and Graham W R 1984 J. Vac. Sci. Technol. A2 939

Wei Zh Q, Xia T D, Bai L F, Wang J, Wu Zh G and Yan P X 2006 Mater. Lett. 60766

Yu X F, Liu X, Zhang K and Hu Z Q 1999 J. Phys. Condens. Matter. 11937

Zheng H G, Liang J H, Zeng J H and Qian Y T 2001 Mater. Res. Bull. 36947 